\title{
PALINOMORFOS MIOCENOS DEL SUBSUELO DE SANTIAGO DEL ESTERO, ARGENTINA
}

\author{
EDUARDO G. OTTONE ${ }^{1}$, SARA M.E. REINARTE MAZURIER ${ }^{2}$ Y ALICIA SALINAS ${ }^{2}$
}

\begin{abstract}
${ }^{1}$ Instituto de Estudios Andinos Don Pablo Groeber-CONICET, Departamento de Ciencias Geológicas, Facultad de Ciencias Exactas y Naturales, Universidad de Buenos Aires, Pabellón n², Ciudad Universitaria, C1428EHA, Buenos Aires, Argentina. ottone@gl.fcen.uba.ar

2 YPF S.A., Gerencia Nuevas Cuencas, Dirección de Exploración, Av. Macacha Güemes 515, Puerto Madero, C1106BKK Buenos Aires, Argentina. sreinantem@ypf.com; asalinase@ypf.com
\end{abstract}

Resumen. La asociación palinológica recuperada del pozo YPF.SE. LH.x-2 Los Horcones, provincia de Santiago del Estero, está dominada por Amaranthaceae/ Chenopodioideae y algas verdes continentales, con Nothofagaceae y Malvaceae subordinadas. En la parte superior de la sección muestreada hay también dinoflagelados y microforaminíferos marinos. La asociación se habría desarrollado en un ambiente lacustre. El cuerpo de agua habría estado rodeado por una vegetación mayormente hebáceo-arbustiva. El clima habría sido templado-cálido y relativamente árido. La palinoflora estaría relacionada con la ingresión marina que habría afectado el noroeste argentino durante el Mioceno medio (15-13 Ma).

Palabras clave. Argentina. Mioceno. Palinomorfos. Transgresión marina.

Abstract. SUBSURFACE MIOCENE PALYNOMORPHS FROM SANTIAGO DEL ESTERO, ARGENTINA. The palynological assemblage recovered from the YPF.SE. LH.x-2 Los Horcones well, Santiago del Estero Province, is dominated by Amaranthaceae/ Chenopodioideae and freshwater green algae, together with subordinate Nothofagaceae and Malvaceae. Marine dinoflagellates and foraminiferal linnings are also present in the upper half of the sampled section. This assemblage may have developed in a lacustrine environment. A mostly herbaceaous and shrubby vegetation may have surrounded the water-body. The climate may have been warm-temperate and relatively arid. The palynoflora could be related to the marine ingression that affected northwestern Argentina during the middle Miocene (15-13 My).

Keywords. Bioconcretions. Argentina. Miocene. Palynomorphs. Marine transgression.

LA discusión sobre el alcance de las transgresiones marinas del Atlántico durante el Mioceno en el centro oeste y noroeste del país es de larga data (Malumián, 1970; Boltovskoy, 1979; Ramos y Alonso, 1995; Pérez et al., 1996; Hernández et al., 2005; Torra, 2005). En los últimos años se ha sugerido la existencia de tres eventos (Hernández et al., 2005, Marengo 2006). Hacia el Oligoceno tardío?/ Mioceno temprano (ca. 25-20 $\mathrm{Ma})$, un evento transgresivo habría ocupado buena parte de las llanuras Chaqueña y Pampeana. El mismo fue reconocido fundamentalmente en subsuelo y está datado por foraminíferos, ostrácodos y nanoplancton calcáreo (Marengo, 2006).

En el Mioceno medio (15-13 Ma), otra ingresión habría avanzado hasta alcanzar, sin sobrepasarlos, los altos de Michicola y Asunción en el norte de la Argentina, que parecen siempre haber permanecido como elementos positivos (Fig. 1). Este evento se encuentra bien documentado en el este de la Argentina y Uruguay (Aceńolaza, 2000). En el centro y noroeste del territorio se conocen dinoflagelados y microforaminíferos marinos en la Formación Chinches, Cordillera Frontal, provincia de San Juan (Ottone et al., 1998), unidad datada por magne- toestratigrafía en 15-12 Ma (Jordan et al., 1996). En niveles equivalentes de la "Serie del Yeso", provincia de San Juan (Barreda et al., 1998), y la Formación Cerro Morado, provincia de La Rioja (Barreda et al., 2003), hay dinoflagelados que podrían relacionarse con medios marinos o continentales. En la Formación Anta, Valles Calchaquíes, provincia de Salta, hay peces, ostrácodos y foraminíferos, pero éstos podrían referirse a un medio salobre hasta costero (Russo y Serraiotto, 1978, Cione et al., 1995) y además, la asociación palinológica recuperada en la unidad no incluye elementos marinos (Quattrocchio et al., 2003).

En el Mioceno tardío (10-5? Ma) hubo otra transgresión. Este evento está bien documentado y datado en la Península Valdés, provincia de Chubut (Scasso et al., 2001). Sin embargo, y contrariamente a lo que se ha sugerido (Hernández et al., 2005), no hay evidencias convincentes sobre su llegada al noroeste del país. Las faunas de peces, gastrópodos, bivlavos y foraminíferos rotálidos de las formaciones San José y Chiquimil del Grupo Santa María, aflorante en los Valles Calchaquíes, en las provincias de Catamarca, Salta y Tucumán, son 
formas de agua dulce a salobre (Morton, 1984, 1986; Zabert, 1984; Cione 1986) y, en el caso específico de los foraminíferos calcáreos, se ha postulado incluso que podrían haber sido llevados desde la costa hacia el continente por aves migratorias (Gavriloff y Arce, 2010). Por otro lado, las palinofloras de las formaciones Chiquimil (Mautino, 2007, 2011; Mautino y Anzótegui, 1998, 2000, 2002a,b; Mautino et al., 2004) y Palo Pintado (Cuadrado y Anzótegui, 1992; Anzótegui y Cuadrado, 1996; Acevedo et al., 1997; Starck y Anzótegui, 2001), no incluyen elementos marinos.

La palinoflora del pozo Los Horcones x-2, ubicado en el noroeste de la provincia de Santiago del Estero, brinda nuevas evidencias acerca de la presencia eventual de ingresiones marinas miocenas en la región (Fig. 1).

\section{MATERIALES Y MÉTODOS}

En esta contribución se analizaron cinco muestras de cut- ting provenientes del sondeo exploratorio YPF.SE. LH.x-2 Los Horcones, distante unos $20 \mathrm{~km}$ de la localidad de Nueva Esperanza, aproximadamente a $26^{\circ} 10^{\prime} \mathrm{S}-64^{\circ} 10^{\prime} \mathrm{O}$, provincia de Santiago del Estero (Fig. 1).

Sólo se dispuso de cinco muestras para llevar adelante este trabajo y si bien es cierto que una mayor densidad de muestreo habría posibilitado lograr conclusiones más precisas en lo que hace a la interpretación de la asociación palinológica, los resultados alcanzados son relevantes y es por eso que se dan a conocer. Las muestras palinológicas fueron procesadas de acuerdo a prácticas convencionales. Carbonatos y silicatos se removieron por tratamiento con ácidos clorhídrico y fluorhídrico a temperatura ambiente. Primero se trató la roca con $\mathrm{HCl}(20 \%)$ y luego de sucesivos lavados, con HF (40\%), hasta lograr el consumo total de la fracción silícea. El residuo orgánico se lavó hasta su neutralización y, previo filtrado con mallas de 25 y $10 \mu \mathrm{m}$, se montó en gelatina-glicerina. Los

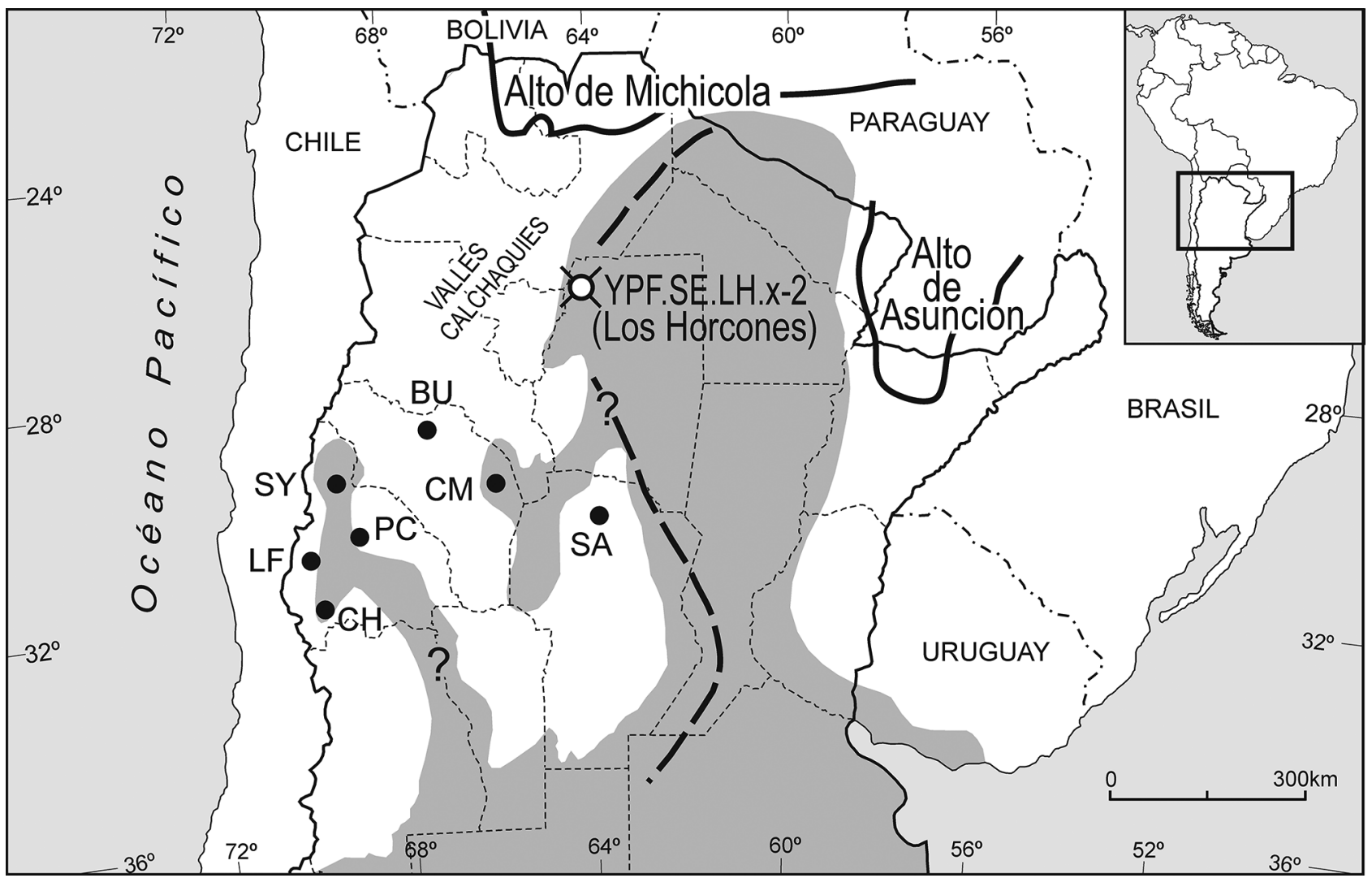

Figura 1. En gris, distribución tentativa de la ingresión marina del Mioceno medio (15-13 Ma) en el norte de la Argentina. Basado en las reconstrucciones de Pérez et al. (1996) y Hernández et al. (2005). La línea de trazo grueso y discontinuo representa el límite oriental de la transgresión sugerido por Ruskin et al. (2011). Los signos de interrogación indican las zonas donde la presencia de la transgresión está en discusión. Las líneas contínuas de trazo grueso limitan zonas de paleorelieve positivo. Ubicación del pozo YPF.SE. LH.x-2 Los Horcones y las localidades donde afloran diferentes unidades citadas en el texto, tales como las formaciones Chinches (CH), Las Flores (LF), Pachaco (PC), del Abra y del Buey (BU), Cerro Morado (CM), Saigún (SA) y la "Serie del Yeso" (SY), así también como las formaciones Anta, San José, Chiquimil y Palo Pintado, con afloramientos en los Valles Calchaquíes. 
especímenes se examinaron con un microscopio binocular Leitz Orthoplan, en tanto que las fotomicrografías se realizaron con una cámara digital Sony Cyber-shot DSC-P93A. Los preparados palinológicos llevan el prefijo BAFC-Pl. El cutting recuperado de los 952-955 metros bajo boca de pozo (mbbp)

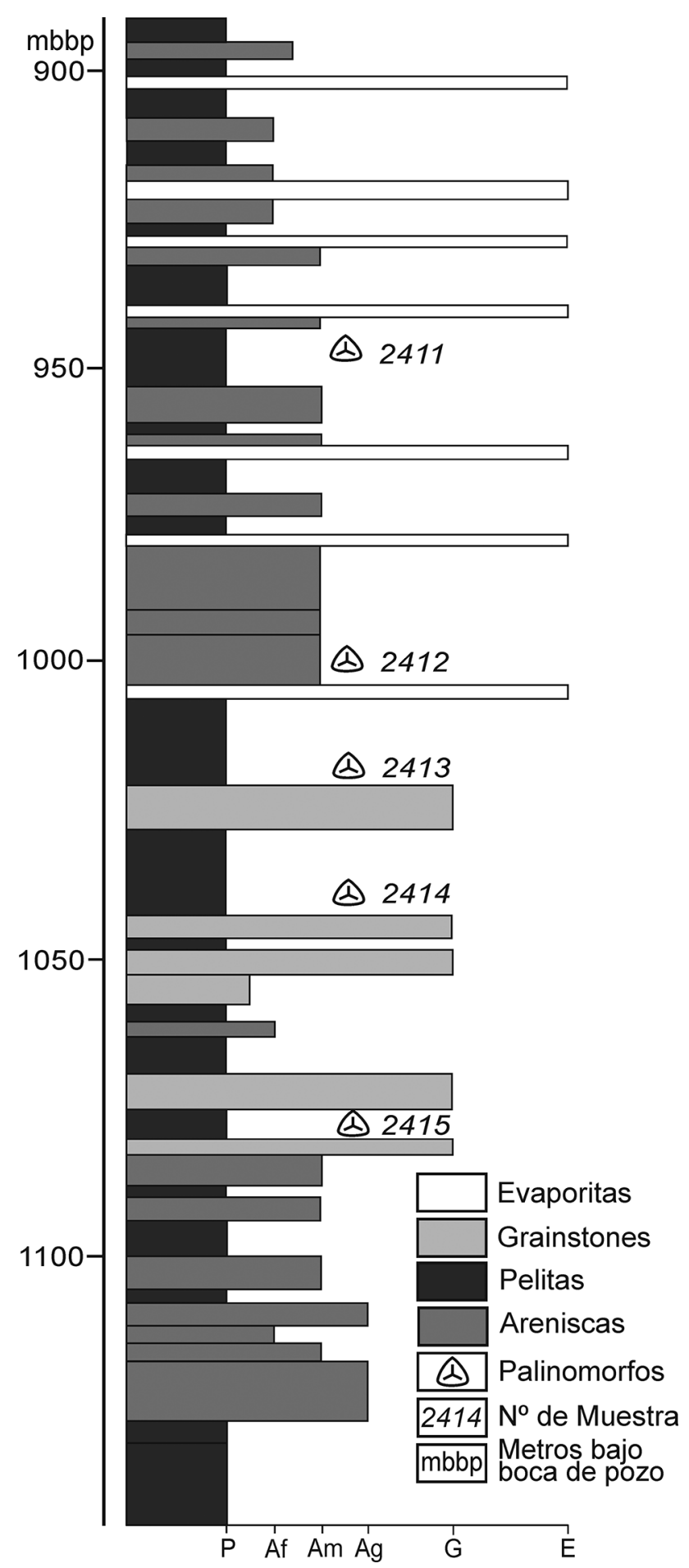

Figura 2. Sección esquemática del pozo Los Horcones $x-2$. corresponde al BAFC-Pl 2411, el de 1000-1003 mbbp al BAFC-Pl 2412, el de 1018-1021 mbbp al BAFC-Pl 2413, el de 1045-1046 mbbp al BAFC-Pl 2414 y el de 1072-1075 mbbp al BAFC-Pl 2415. Los especímenes ilustrados están identificados con el número de preparado y las referencias de England Finder. Los preparados se encuentran alojados en el Departamento de Ciencias Geológicas, Facultad de Ciencias Exactas y Naturales, Universidad de Buenos Aires.

Para confeccionar la Tabla 1 se realizaron conteos sobre el número total de palinomorfos presentes por preparado. Teniendo en cuenta que la confiabilidad de los conteos porcentuales disminuye cuando, como en este caso, los especímenes disponibles son relativamente escasos, se presentan los datos a modo de porcentajes que sólo pretenden ser orientativos acerca de la composición de la palinoflora. En la Tabla 1 los taxones aparecen listados por orden alfabético en cada una de las muestras y entre muestras sucesivas por orden de aparición.

E.G. Ottone es responsable del estudio paleontológico, en tanto que S.M.E. Reinarte Mazurier y A. Salinas realizaron la parte geológica.

\section{GEOLOGÍA}

La información geológica disponible sobre la sucesión sedimentaria atravesada por el pozo Los Horcones es muy limitada. Las sedimentitas neógenas alcanzan un espesor de aproximadamente $1100 \mathrm{~m}$ y se apoyan discordantemente sobre el Devónico de la Formación Rincón. El intervalo estratigráfico que incluye los horizontes fosilíferos es mayormente pelítico. El análisis del cutting recuperado refleja la presencia de una sucesión clástica a calcáreo-evaporítica compuesta principalmente por pelitas gris verdosas y, en menor proporción, areniscas cuarzosas de moderada selección, junto a grainstones oolítico arenosos, yeso nodular y tobas subordinadas (Fig. 2). Las evaporitas sugerirían un cuerpo de agua somero asociado a condiciones relativamente áridas.

\section{PALINOLOGÍA}

Los palinomorfos provienen del tramo 952-955 a 10721077 mbbp del pozo Los Horcones x-2 (Tab. 1). La asociación es poco abundante, muestra baja diversidad y está mayoritariamente compuesta por elementos de origen continental con escasas formas marinas presentes hacia la parte cuspidal, en los niveles 952-955, 1000-1003 y 1018-1021 mbbp. Los palinomorfos están regularmente preservados. 
La lista de las formas identificadas se da a continuación. Para el ordenamiento taxonómico de las estreptofitas, se siguieron distintas propuestas clasificatorias: la de Crandall-Stotler y Stotler (2000) para las briófitas, la de Smith et al. (2006) para los helechos, la de Christenhusz et al. (2011) para las gimnospermas y las de Soltis et al. (2005), Bremer et al. (2009) y Reveal (2012) para las angiospermas. En estos trabajos se encuentran las referencias correspondientes a los rangos supraespecíficos citados a continuación. Las afinidades botánicas están a nivel familia y, en Amaranthaceae y Fabaceae, subfamilia.

\section{BRIÓFITAS}

MARCHANTIOPSIDA Crandall-Stotler y Stotler 2000

MARCHANTIALES Limpricht 1876

AYTONIACEAE Cavers 1911

Reboulisporites fuegiensis Zamaloa y Romero 1990

(Fig. 3.19)

\section{MONILOFITAS (HELECHOS)}

POLYPODIOPSIDA (FILICOPSIDA) Cronquist,

Takhtajan y Zimmermann 1966

GLEICHENIALES Link 1833

DIPTERIDACEAE Seward y Dale 1901/

GLEICHENIACEAE Presl 1825

Laevigatosporites sp. (Fig. 3.15)

SALVINIALES Link 1833

SALVINIACEAE Martynov 1820

Azolla sp. (Fig. 4.2)

CYATHEALES Frank 1877

CYATHEACEAE Kaulfuss 1827/ DICKSONIACEAE

Schomburgk 1848

Deltoidospora sp. (Figs. 4.4)

POLYPODIALES Link 1833
PTERIDACEAE Ching 1975

Muricingulisporis chenquensis Barreda 1992 (Fig. 4.3)

GIMNOSPERMAS

GNETIDAE Pax 1894

EPHEDRALES Dumortier 1829

EPHEDRACEAE Dumortier 1829

Equisetosporites claricristatus (Shakmundes) Barreda 1997a (Fig. 3.10)

Equisetosporites notensis (Cookson) Romero 1977

(Fig. 3.11)

PINIDAE Cronquist, Takhtajan y Zimmermann 1966 ARAUCARIALES Gorozhankin 1904 PODOCARPACEAE Endlicher 1847

Lygistepollenites florinii (Cookson y Pike) Stover y Evans 1973 (Fig. 4.6)

Phyllocladidites mawsonii Cookson 1947 (Fig. 3.12)

Podocarpidites marwickii Couper 1953 (Fig. 4.1)

ANGIOSPERMAS MONOCOTILEDÓNEAS

LILIALES Perleb 1826

LILIACEAE de Jussieu 1789

Liliacidites sp. (Fig. 3.13)

POALES Small 1903

POACEAE Barnhart 1895

Graminidites sp. (Fig. 3.5)

CORE-EUDICOTILEDÓNEAS

CARYOPHYLLALES Perleb 1826

AMARANTHACEAE de Jussieu 1789

CHENOPODIOIDEAE Burnett 1835

Chenopodipollis chenopodiaceoides (Martin) Truswell en Truswell et al. 1985 (Fig. 3.3)

Figura 3. 1-2, Tricolpites trioblatus Mildenhall y Pocknall 1989; 1, BAFC-PI 2413(4) P38/ 3; 2, BAFC-PI 2413(4) H22/ 3; 3, Chenopodipollis chenopodiaceoides (Martin) Truswell en Truswell et al. 1985, BAFC-PI 2413(4) P53/ 0; 4, Nothofagidites dorotensis Romero 1973, BAFC-PI 2413(4) N43/ 1; 5, Graminidites sp., BAFC-PI 2413(8) K23/ 0; 6, Nothofagidites saraensis Menéndez y Caccavari de Fílice 1975, BAFC-PI 2412(4) G31/ 4; 7, Nothofagidites fuegiensis Menéndez y Caccavari de Fílice 1975, BAFC-PI 2413(4) Q47/ 4; 8, Tricolpites sp. BAFC-PI 2413(4) H31/ 1; 9, Margocolporites vanwijhei Gemeraad, Hopping y Müller 1968, BAFC-PI 2414(6) R32/ 0; 10, Equisetosporites claricristatus (Shakmundes) Barreda 1997a, BAFC-PI 2412(4) X44/ 4; 11, Equisetosporites notensis (Cookson) Romero 1977, BAFC-PI 2414(6) K34/ 4; 12, Phyllocladidites mawsonii Cookson 1947, BAFC-PI 2412(3) F15/ 3; 13, Liliacidites sp., BAFC-PI 2413(4) A23/ 1; 14, Senipites sp. cf. S. patagonica Barreda 1997b, BAFC-PI 2414(6) R32/ 0; 15, Laevigatosporites sp., BAFC-PI 2412(4) J36/ 2; 16, Echiperiporites sp?, BAFC-PI 2413(4) 51E/ 0; 17, Acaciapollenites sp. cf. A. myriosporites Mildenhall 1972, BAFC-PI 2411(3) M51/ 3; 18, Polliadopollenites sp., BAFC-PI 2414(3) A22/ 2; 19, Reboulisporites fuegiensis Zamaloa y Romero 1990, BAFC-PI 2413(1) S35/ 09. Escala gráfica $=10 \mu \mathrm{m}$. 
(1)

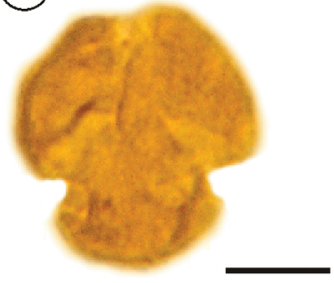

(5)

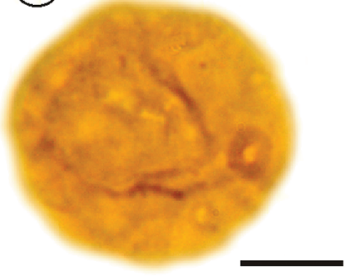

(9)

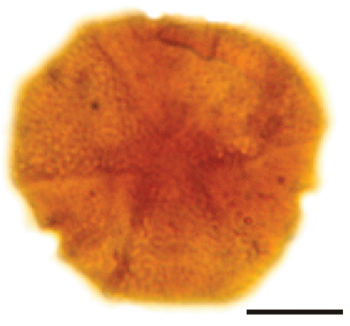

(13)

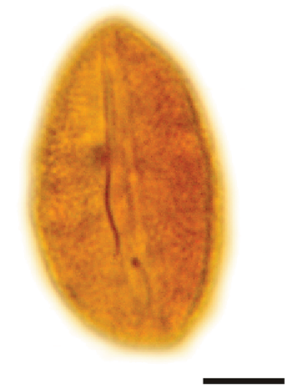

(17)

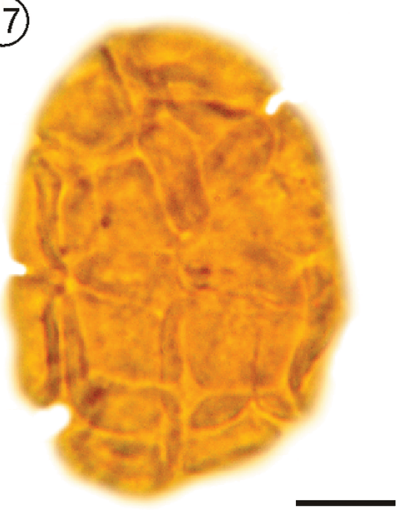

(2)

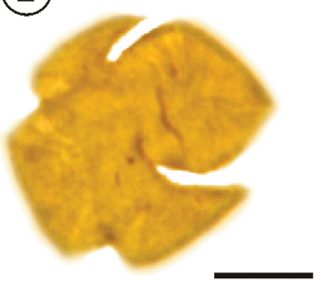

(6)

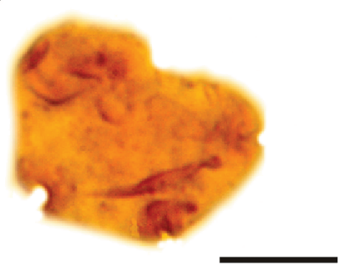

(10)

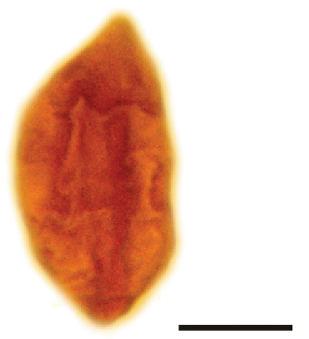

(14)

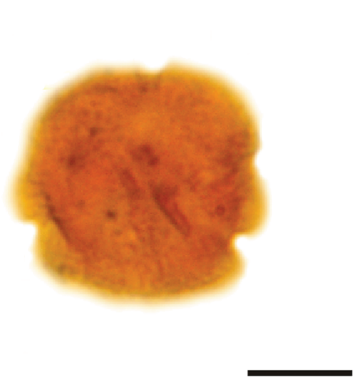

(18)
(3)

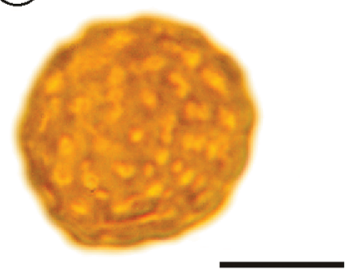

(7)

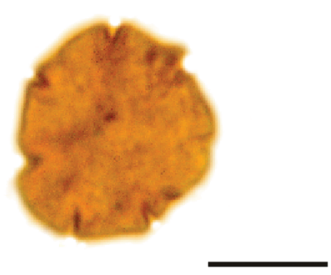

(11)

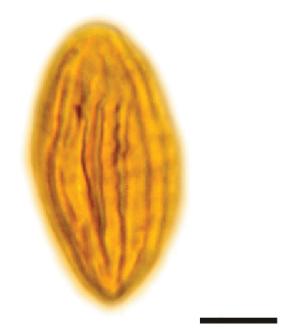

(15)

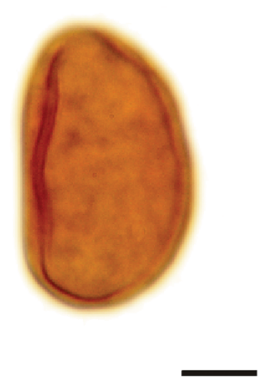

(4)

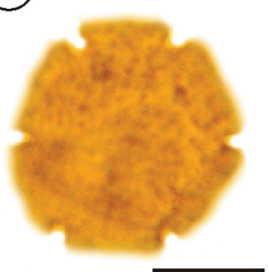

(8)

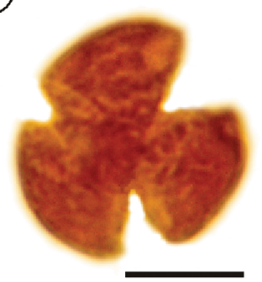

(12)

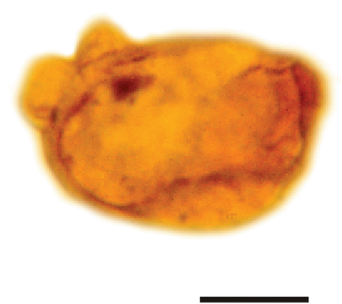

(16)

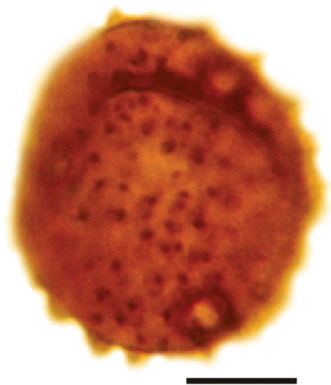

(19)
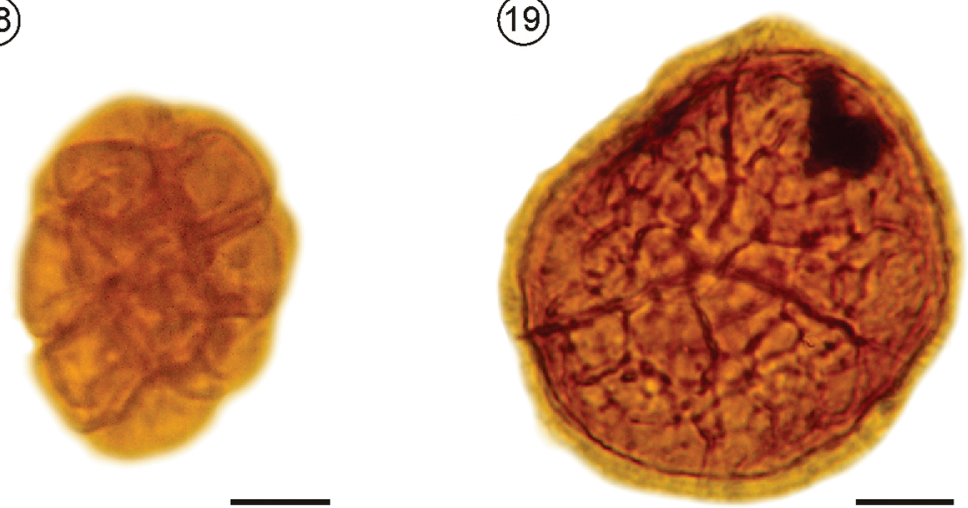
(1)

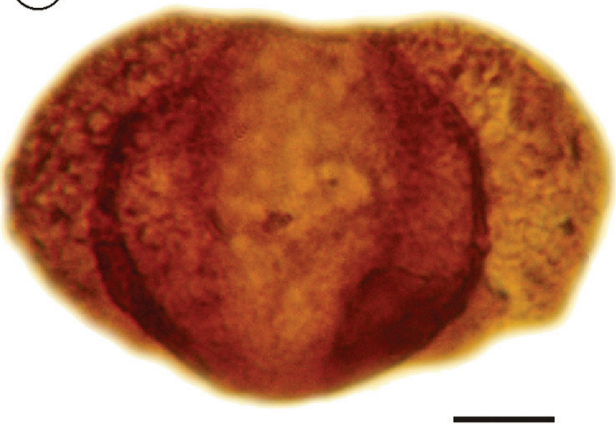

(2)

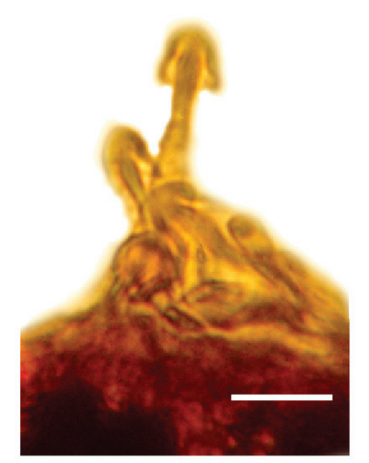

(4)

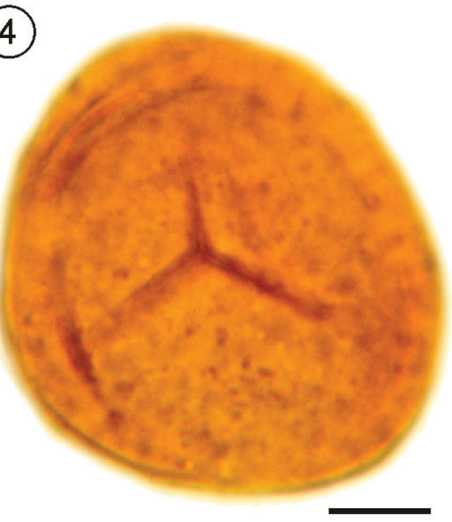

(7)

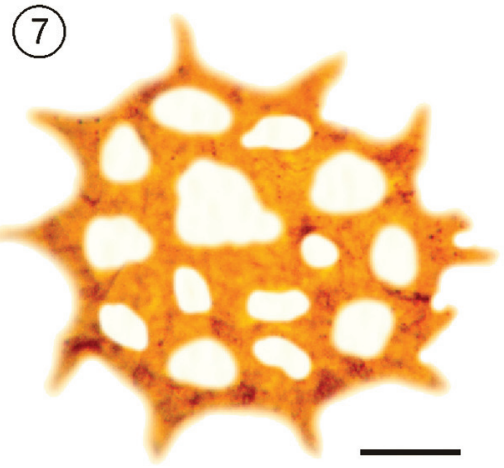

(10)

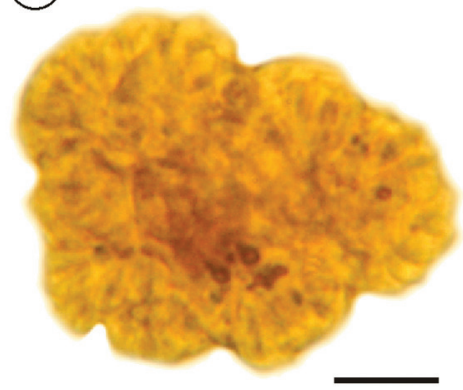

(5)

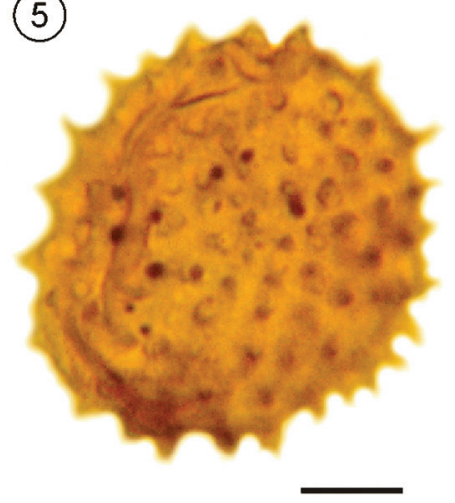

(8)

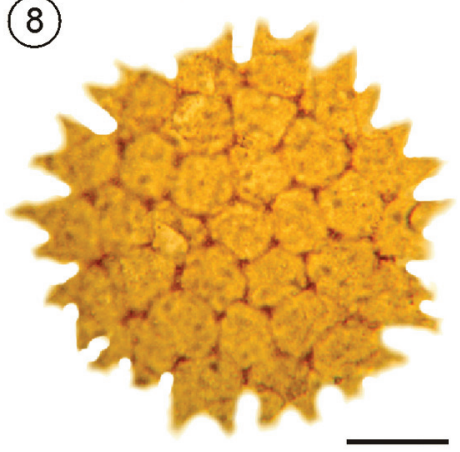

(11)

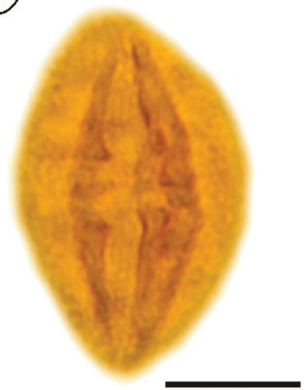

(3)

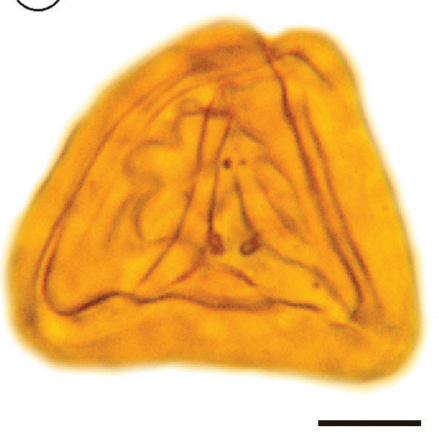

(6)

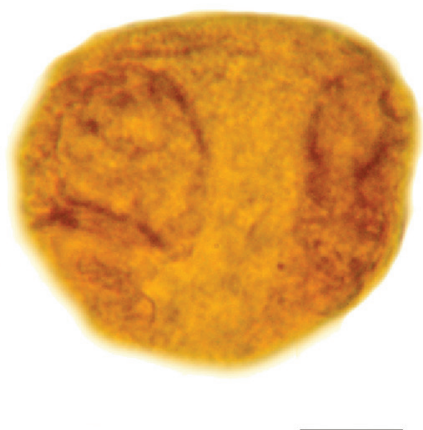

(9)

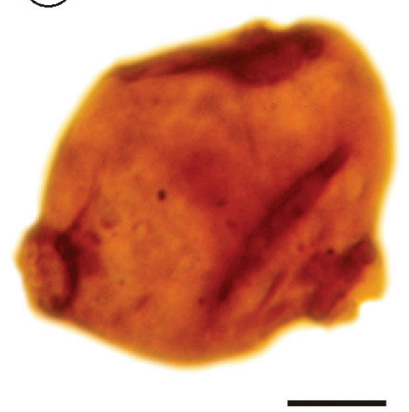

(12)

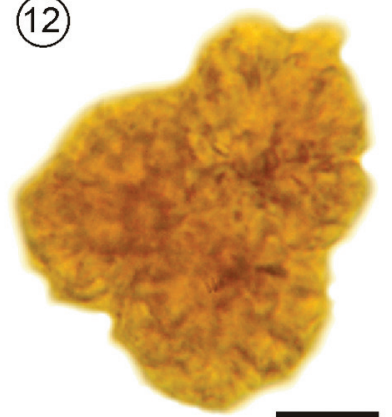


ROSIDES

MYRTALES Reichenbach 1828

ONAGRACEAE de Jussieu 1789

Corsinipollenites sp. cf. C. atlantica Barreda 1997c

(Fig. 4.9)

\section{EUROSIDES I (FÁBIDAS)}

FABALES Bromhead 1838

FABACEAE Lindley 1836

CAESALPINIACEAE Brown 1814

Margocolporites vanwijhei Gemeraad et al. 1968

(Fig. 3.9)

LEGUMINOSAE de Jussieu 1789

Acaciapollenites sp. cf. A. myriosporites Mildenhall 1972 (Fig. 3.17)

Polliadopollenites sp. (Fig. 3.18)

FAGALES Engler 1892

NOTHOFAGACEAE Kuprian 1962

Nothofagidites dorotensis Romero 1973 (Fig. 3.4)

Nothofagidites fuegiensis Menéndez y Caccavari de

Fílice 1975 (Fig. 3.7)

Nothofagidites saraensis Menéndez y Caccavari de

Fílice 1975 (Fig. 3.6)

EUROSIDES II (MÁLVIDAS)

MALVALES Dumortier 1829

MALVACEAE de Jussieu 1789

Echiperiporites sp? (Figs. 3.16)

Malvacipolloides sp? (Figs. 4.5)

ASTÉRIDAS

ERICALES Dumortier 1829

SYMPLOCACEAE Desfontaine 1820

Senipites sp. cf. S. patagonica Barreda 1997b

(Fig. 3.14)
EUASTÉRIDAS I (LÁMIDAS)

SOLANALES Dumortier 1829

CONVOLVULACEAE de Jussieu 1789

Tricolpites trioblatus Mildenhall y Pocknall 1989

(Fig. 3.1-2)

DICOTILEDÓNEAS DE AFINIDAD INCIERTA

Rhoipites sp. (Figs. 4.11)

Tricolpites sp. (Fig. 3.8)

\section{CLORÓFITAS CONTINENTALES}

Botryococcus sp. (Fig. 4.10-12)

Monactinus sp. cf. M. simplex (Meyen) Corda 1839

(Fig. 4.7)

Pseudopediastrum sp. cf. P. boryanum (Turpin)

Hegewald en Buchheim et al. 2005 (Fig. 4.8)

PRASINOFITAS

Pterospermella sp.

DINOFLAGELADOS

Hytrichosphaeridium sp?

Operculodinium sp? (Fig. 5.2)

Spiniferites sp. (Fig 5.3)

\section{MICROFORAMINÍFEROS (Fig. 5.1)}

El material recuperado se considera en líneas generales autóctono en tanto no se observan diferencias notorias de preservación entre las formas presentes, aunque, tratándose de cutting, no es descartable la presencia de contaminación intraformacional producida por caving durante la perforación. De acuerdo a los conteos porcentuales, resulta evidente que la forma mejor representada es Chenopodipollis chenopodiaceoides, Amaranthaceae/ Chenopodioideae, y, aunque están au-

Figura 4. 1, Podocarpidites marwickii Couper 1953, BAFC-PI 2413(4) U38/ 2; 2, Azolla sp., BAFC-PI 2413(1) O43/ 0, detalle de los gloquidios; 3, Muricingulisporis chenquensis Barreda 1992, BAFC-PI 2412(3) O13/ 0; 4, Deltoidospora sp., BAFC-PI 2413(7) V37/ 3; 5, Malvacipolloides sp?, BAFC-PI 2414(3) G69/ 0; 6, Lygistepollenites florinii (Cookson y Pike) Stover y Evans 1973, BAFC-PI 2415(6) K43/ 2; 7, Monactinus sp. cf. M. simplex (Meyen) Corda 1839, BAFC-PI 2413(2) M51/ 3; 8, Pseudopediastrum sp. cf. P. boryanum (Turpin) Egewald en Bucheim et al. 2005, BAFC-PI 2413(1) B52/ 2; 9, Corsinipollenites sp. cf. C. atlantica Barreda 1997c, BAFC-PI 2413(8) F43/ 3; 10, 12, Botryococcus sp., 10, BAFC-PI 2413(7) M51/ 3, 12, BAFC-PI 2413(3) H37/ 2; 11, Rhoipites sp., BAFC-PI 2414(6) K35/ 1. Escala gráfica/ Scale bar = $10 \mu \mathrm{m}$, con la excepción de 7, $10=20 \mu \mathrm{m}$. 


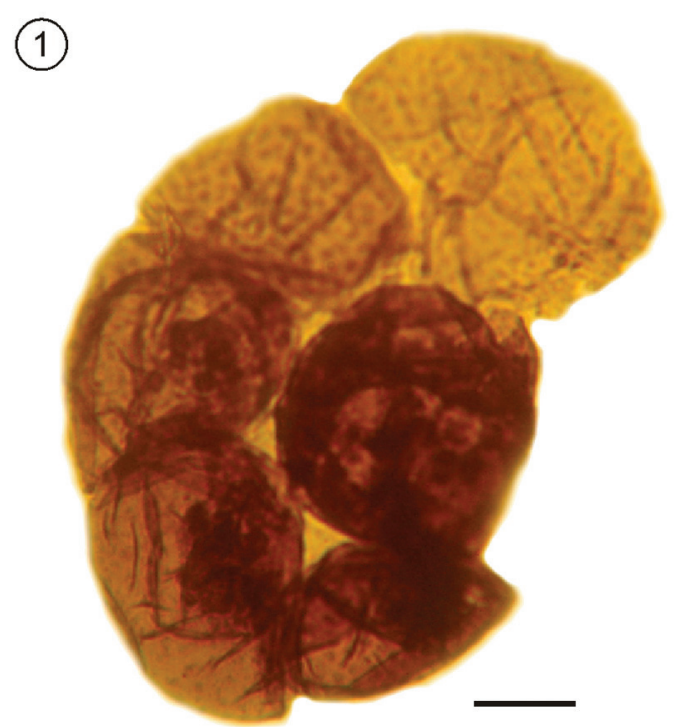

(2)

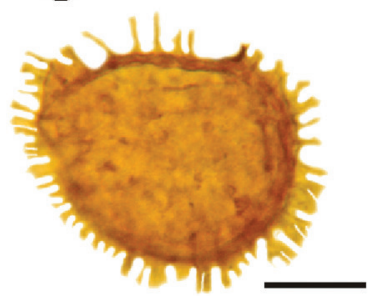

(3)

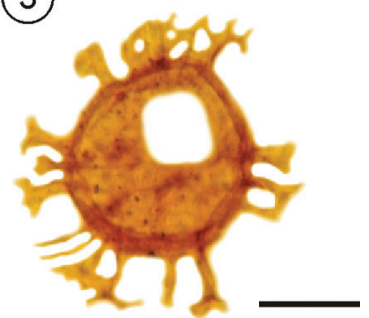

Figura 5. 1, Microforaminífero, BAFC-PI 2413(1) A32/ 0; 2, Operculodinium sp?, BAFC-PI 2412(5) G54/ 1; 3, Spiniferites sp., BAFC-PI 2412(2) $D 23 / 3$. Escala gráfica $=20 \mu \mathrm{m}$.

sentes en el nivel cuspidal de la sección analizada, las algas de agua dulce Botryococcus sp. y Pseudopediastrum sp. cf. P. boryanum (Tab. 1). Otras formas relativamente bien representadas son las Nothofagaceae, Rhoipites sp. y, en menor medida, las Malvaceae y las Podocarpaceae, en especial Podocarpidites marwickii. El resto de las especies, incluídas las marinas, muestran en general escasa representación, llegando a darse el caso de alguna de ellas, como Corsinipollenites sp. cf. C. atlantica, de la que sólo se ha recuperado un único especímen.

\section{PALEOAMBIENTE}

La asociación parece reflejar la presencia de un cuerpo de agua dulce a salobre con abundante fitoplancton y plantas como las Salviniaceae. Botryococcus muestra una amplia distribución geográfica y se da tanto en medios eutróficos como oligotróficos (Komárek y Marvan, 1992). Monactinus simplex y Pseudopediastrum boryanum también se encuentran en latitudes diversas, aunque son característicos de reservorios eutrófi- cos a mesotróficos de agua dulce neutral a alcalina, y en el caso de $P$. boryanum, puede también progresar en aguas mesohalinas (Komárek y Jankovská, 2001). Las Salviniaceae son plantas flotadoras que progresan en medios dulceacuícolas estancados o de poco movimiento (Collinson, 2002).

Este cuerpo de agua habría estado rodeado por vegetales halófitos hasta xerófitos como las Amaranthaceae/ Chenopodioideae, Convolvulaceae, Ephedraceae y Malvaceae (Frederiksen, 1985; Martin, 1989, 2000; Mautino, 2011). Algo alejada probablemente del reservorio habría progresado una comunidad arbórea dominada por Podocarpaceae. Estas plantas producen polen sacado que, disperso por el viento, suele aparecer bien representado a larga distancia de su flora parental (Markgraf, 1983). Sin embargo, en la actualidad son conspicuas integrantes de algunas comarcas húmedas en la región, por lo que es dable pensar que lo mismo haya sucedido en el Cenozoico.

Es llamativa la presencia de las Nothofagaceae, ya que este grupo de plantas no tenía hasta el momento registros seguros tan septentrionales en el Neógeno sudamericano (Barreda et al., 2007), alcanzando $c a$. de $29^{\circ} 30^{\prime} \mathrm{S}$ en la Argentina y $33^{\circ} 50^{\prime} \mathrm{S}$ en Chile (Barreda et al., 1998, 2003, 2011). El material figurado como Nothofagidites sp. 1 para el Mioceno tardío/ Plioceno de Brasil, ca. 22 45' S (Garcia et al., 2008), está bastante mal preservado por lo que no muestra claramente los caracteres diagnósticos de este tipo polínico. Nothofagus es actualmente un árbol autóctono de los bosques subantárticos de la Patagonia andina, sin embargo, su polen suele aparecer a gran distancia, por ejemplo en Mar del Plata, Mar Chiquita, Magdalena y La Plata, provincia de Buenos Aires (Majas y Romero, 1992; Gassmann y Pérez, 2006a, b; Nitiu, 2009; Latorre et al., 2010). Estas localidades se encuentran a más de $1500 \mathrm{~km}$ al noroeste de la zona de aporte. Por lo tanto, no es de descartar que una situación semejante se haya dado en el pasado. Sin embargo, fuera de América del Sur, Nothofagus es autóctono de Nueva Guinea, una isla emplazada al norte de Australia en una latitud subecuatorial (Knapp et al., 2005), lo que demuestra que estas plantas pueden también adaptarse a hábitats más cálidos que los que ocupan en nuestro país.

Otro punto que llama la atención es la falta de polen de Asteraceae en la asociación, ya que es un elemento típico de las palinofloras neógenas de la Argentina (Barreda et al., 2007). Este hecho podría deberse a la ausencia de estos vegetales en el medio. Sin embargo, hay que tener en cuenta que muchos gru- 
TABLA 1 - Distribución de los palinomorfos en el pozo YPF.SE.LH.x-2 Los Horcones. A, abundante (más de 10\% del contenido total de palinomorfos); C, común (5-10\%); E, escaso (1-5\%); $R$, raro (menos de 1\%).

\begin{tabular}{|c|c|c|c|c|c|}
\hline & 2411 & 2412 & 2413 & 2414 & 2415 \\
\hline \multicolumn{6}{|l|}{ Miosporas } \\
\hline Acaciapollenites sp. cf. A. myriosporites & $R$ & & & & \\
\hline Chenopodipollis chenopodiaceoides & $A$ & $A$ & $A$ & $A$ & $A$ \\
\hline Malvacipolloides sp.? & $E$ & $E$ & $E$ & $E$ & $E$ \\
\hline Nothofagidites saraensis & C & C & & C & $C$ \\
\hline Rhoipites sp. & C & C & C & C & $C$ \\
\hline Azolla sp. & $E$ & $E$ & $E$ & & $E$ \\
\hline Deltoidospora sp. & & $E$ & $E$ & & \\
\hline Graminidites sp. & & $E$ & $E$ & & $E$ \\
\hline Laevigatosporites sp. & & $E$ & $E$ & & \\
\hline Muricingulisporis chenquensis & & $E$ & $E$ & & \\
\hline Phyllocladidites mawsonii & & $R$ & & & \\
\hline Podocarpites marwickii & & $E$ & $E$ & E & \\
\hline Reboulisporites fuegiensis & & $E$ & $E$ & $E$ & \\
\hline Corsinipollenites sp. cf. C. atlántica & & & $R$ & & \\
\hline Echiperiporites sp. & & & $R$ & $R$ & \\
\hline Liliacidites sp. & & & $R$ & & \\
\hline Nothofagidites fuegiensis & & & C & & $C$ \\
\hline Senipites sp. cf. S. patagonica & & & $R$ & $R$ & \\
\hline Tricolpites trioblatus & & & $E$ & $E$ & \\
\hline Tricolpites sp. & & & $E$ & $E$ & \\
\hline Equisetosporites notensis & & & & $R$ & \\
\hline Margocolporites vanwijhei & & & & $R$ & \\
\hline Poliadopollenites sp. & & & & $R$ & \\
\hline Lygistepollenites florinii & & & & $R$ & $R$ \\
\hline Nothofagidites dorotensis & & & & & $E$ \\
\hline Proteacidites sp. & & & & & $R$ \\
\hline
\end{tabular}

\section{Algas continentales}

\begin{tabular}{|lllll}
\hline Botryococcus sp. & $A$ & $A$ & $A$ & $A$ \\
\hline Monactinus sp. $\boldsymbol{c f}$. M. simplex & & $R$ & & $A$ \\
\hline Pseudopediastrum sp. cf. P. boryanum & $A$ & $A$ & $A$
\end{tabular}

Pterospermella sp.

$R \quad R$

\section{Dinoflagelados}

Hystrichosphaeridium sp.?

Operculodinium sp.?

Spiniferites sp.

\section{Microforaminíferos}

Indeterminados $E \quad E$

pos son muy escasos en la palinoflora de Los Horcones y, tal como se ha mencionado anteriormente, están en ocasiones representados por un único espécimen, por lo que la falta de polen de Asteraceae no implica necesariamente que el grupo no haya formado parte de la flora parental.

En este ambiente parece haberse producido una ingresión marina, acotada a los tramos cuspidales de la sección muestreada. Entre los elementos marinos se destacan dinoflagelados y microforaminíferos. El dinoflagelado gonyaulacoide Spiniferites sp. resulta ambiguo en el momento de la evaluación paleoambiental. Esta forma no parece poseer elementos que, como las trabéculas, indicarían, en quistes marinos del 
complejo Gonyaulax spinifera (Kofoid, 1911), un cierto nivel de estrés ambiental debido a variaciones en la salinidad (Rochon et al., 2009). Sin embargo, un dinoquiste como S. cruciformis Wall et al. 1973, a pesar de poseer muros y espinas gonales trifurcadas sin trabéculas, podría estar relacionado a medios de agua dulce (Kouli et al., 2001). A nivel mundial (Ruskin et al., 2011) y en nuestro país en particular (Boltovskoy, 1979; González et al., 1980; Laprida et al., 2009), está bien documentada la presencia de foraminíferos en cuerpos de agua dulce o salina desconectados del mar. Sin embargo, en lo que hace específicamente a los microforaminíferos, no hay citas ciertas en medios que no sean marinos o con influencia marina (Stancliffe, 1996; Zamora et al., 2007).

El registro de grupos de distribución tropical como Caesalpiniaceae, Leguminosae y Symplocaceae sugeriría un clima templado cálido (Heywood, 1993; Richards, 1998), en tanto que, tal como ya fuera referido, la presencia de evaporitas indicaría que el mismo fue también relativamente árido.

\section{EDAD Y CORRELACIONES}

Desde un punto de vista bioestratigráfico la asociación incluye formas como Acaciapollenites sp. cf. A. myriosporites, Chenopodipollis chenopodiaceoides, Corsinipollenites sp. cf. C. atlantica, Polliadopollenites sp., Senipites sp. cf. S. patagonica y Tricolpites trioblatus, que tienen sus primeros registros hacia el Oligoceno cuspidal?/ Mioceno basal (Barreda, 1997a,b,c; Barreda y Caccavari, 1992). Estos elementos aparecen junto a esporas de briófitas, como Reboulisporites fuegiensis, y Pteridaceae, tal como Muricingulisporis chenquensis, y polen de Podocarpaceae, Ephedraceae, Malvaceae y Nothofagaceae.

El conjunto es comparable con otras palinofloras del Mioceno de la Argentina (Barreda et al., 2007) y, en especial, con aquellas del Mioceno medio del centro oeste y noroeste del país, descriptas para la Formación Chinches y "Serie del Yeso", Cordillera Frontal, provincia de San Juan, y la Formación Cerro Morado, en el ámbito de Precordillera, provincia de La Rioja (Ottone et al., 1998; Barreda et al., 1998, 2003) y, probablemente, Pachaco (Prámparo et al., 1996), y también para la Formación del Buey, Sierra de Famatina (Barreda et al., 2006) y la Formación San José, Valles Calchaquíes (Mautino, 2007, 2011; Mautino et al., 2012).

La asociación palinológica del Mioceno tardío de las formaciones Chiquimil (Mautino, 2007, 2011; Mautino y Anzótegui, 1998, 2000, 2002a,b; Mautino et al., 2004) y Palo
Pintado (Cuadrado y Anzótegui, 1992; Anzótegui y Cuadrado, 1996; Acevedo et al., 1997; Starck y Anzótegui, 2001), también aflorantes en los Valles Calchaquíes, presentan diferentes tipos morfológicos de esporas, polen y Chlorophyta que las hace, en líneas generales, comparables a la palinoflora de Los Horcones, sin embargo, la primera se distingue por mostrar una mayor variedad de Asteraceae (Mautino y Anzótegui, 2002b), mientras que la segunda presenta cantidades variables de esporas de monilofitas junto a polen de Anacardiaceae, Aquifoliaceae y Sapotaceae (Galli et al., 2011). Otro tanto ocurre con la palinoflora del Mioceno cuspidal de la Formación Las Flores, provincia de San Juan (Prámparo et al., 1998; Bracco et al., 2011). Sin embargo, en ésta última no se han realizado estudios detallados, lo que dificulta su comparación. En estas ascociaciones del Mioceno tardío no hay fósiles marinos.

Resulta difícil comparar los horizontes del pozo Los Horcones $\mathrm{x}-2$ con los de la ingresión marina que afectó la región hacia el Oligoceno tardío?/ Mioceno temprano, ya que en los últimos no hay palinomorfos. De todos modos, cabe acotar que este evento transgresivo muestra en la zona un espesor importante y abundantes microfósiles calcáreos (Marengo, 2006), lo que indicaría un ambiente de depositación distinto al aquí descripto.

Este conjunto de datos sugeriría entonces una edad Mioceno o más joven para la asociación palinológica aquí estudiada. Sin embargo, teniendo en cuenta su composición y la presencia de palinomorfos marinos, y a pesar de no incluir indicadores bioestratigráficos demasiado precisos, la palinoflora de Los Horcones podría referirse, con cierto margen de dudas, al Mioceno medio.

\section{INFERENCIAS PALEOGEOGRÁFICAS}

El evento transgresivo del Mioceno Medio, con el que podría relacionarse la asociación marina de Los Horcones, habría sido importante en el centro y norte del país, incluyendo el sur de Uruguay y parte de Paraguay (Fig. 1).

Estudios de isótopos estables de ${ }^{13} \mathrm{C}$ y ${ }^{18} \mathrm{O}$ llevados a cabo en la Formación Saguión, ESE de Salinas Grandes, provincia de Córdoba, la Formación Anta en la Quebrada de la Yesera y el Valle de Lerma, Valles Calchaquíes, provincia de Salta, las formaciones del Buey y del Abra en la Sierra de Famatina, provincia de La Rioja, y la Formación Chinches en la Cordillera Frontal de San Juan, sugerirían un origen lacustre para estas 
unidades. En concordancia con estos datos, el límite occidental de la transgresión miocena en el noroeste del país estaría aproximadamente en los $65^{\circ} \mathrm{O}$ (Ruskin et al., 2011) (Fig. 1).

La evidencia acerca del alcance de la transgresión del Mioceno medio en el centro oeste y noroeste del país resulta entonces contradictoria. En la Formación Cerro Morado y en la "Serie del Yeso" hay dinoflagelados que, a falta de estudios sistemáticos precisos, podrían considerarse marinos o eventualmente continentales (Barreda et al., 1998, 2003) (Fig. 1). En la Formación Chinches, en afloramientos ubicados hacia el sur de la Quebrada Adelco, Cordón del Espinacito, Cordillera Frontal de San Juan, así como en Los Horcones, hay microforaminíferos (Ottone et al., 1998), y éstos se dan en medios marinos o con influencia marina. Sin embargo, estudios isotópicos parecen indicar un origen continental para los niveles de la cordillera sanjuanina (Fig. 1). Los microforaminíferos de la Formación Chinches podrían entonces ser interpretados: (1) como elementos autóctonos confirmando en ese caso la llegada del mar a la región, por lo que habría que asumir que en el muestreo para isótopos probablemente no se hayan tomado muestras del nivel correspondiente al BAFC-Pl 905, que es el que incluye los microforaminíferos (Ottone et al., 1998); (2) como elementos que, de modo equivalente a lo postulado para foraminíferos calcáreos presentes en medios lacustres, podrían haber sido ocasionalmente llevados por aves migratorias desde la paleocosta del mar hasta el lago (Gavriloff y Arce, 2010), sin embargo, este mecanismo nunca fue descripto para los microforaminíferos; (3) como formas recicladas, aunque esta situación no es sostenible a partir de la observación del material ya que, si bien es cierto que hay elementos reciclados en la asociación, los mismos provendrían del Triásico continental (Ottone et al., 1998).

\section{CONSIDERACIONES FINALES}

A partir del análisis de las asociaciones palinológicas provenientes del pozo Los Horcones se concluye que los niveles portadores se habrían depositado en un medio relativamente estable, con desarrollo de cuerpos de agua dulce a salobre, mayormente eutróficos a mesotróficos, sobre el que se habría producido una ingresión marina. La cubierta vegetal en las cercanías de los reservorios dulceacuícolas habría sido de tipo herbáceo-arbustiva, con desarrollo de formaciones arbóreas algo más alejadas. El clima habría sido templado cálido y relativamente árido.
La palinoflora de Los Horcones podría correlacionarse con asociaciones de edad Mioceno medio presentes en la región. La asociación de dinoflagelados y microforaminíferos marinos se relacionaría con el evento transgresivo neógeno que habría afectado al noroeste argentino entre los 15-13 Ma (Hernández et al., 2005).

En función de la dificultad de determinar con certeza el carácter autóctono o para-autóctono del polen de Nothofagaceae, que es un elemento de extracción austral y muy importante para delimitar las provincias paleo-fitogeográficas de la región, se considera a la microflora de Los Horcones en una zona transicional entre las provincias Neotropical y de Transición (Barreda et al., 2007).

\section{AGRADECIMIENTOS}

Los autores agradecen a G.D. Holfeltz por el procesamiento palinológico de las muestras y a A. González por las ilustraciones. A H. Marengo por facilitarnos la consulta de su Tesis Doctoral. A V. Barreda y un revisor anónimo por sus constructivos comentarios sobre el artículo. A C. Rubinstein (Comité Editor) por sus correcciones y sugerencias. Al Consejo Nacional de Investigaciones Científicas y Técnicas, y a la Universidad de Buenos Aires por las facilidades brindadas. A YPF S.A. por la sesión del material y, en especial, a R. Calegari, Gerente de Nuevas Cuencas y PEA, por habernos permitido dar a conocer estos datos. Esta es la contribución R-107 del Instituto de Estudios Andinos Don Pablo Groeber.

\section{BIBLIOGRAFÍA}

Aceñolaza, F.G. 2000. La Formación Paraná (Mioceno Medio): estratigrafía, distribución regional y unidades equivalentes. Serie Correlación Geológica (INSUGEO) 14: 9-27.

Acevedo, T.R., Mautino, L.R., Anzótegui, M.L. y Cuadrado, G. 1997. Estudio palinológico de la Formación Palo Pintado (Mioceno Superior) provincia de Salta, Argentina. Parte II: esporas. Revista Universidade Guarulhos-Geociências, número especial: 112-120.

Anzótegui, L.M. y Cuadrado, G. 1996. Palinología de la Formación Palo Pintado, Mioceno Superior, provincia de Salta, República Argentina. Parte I: taxones nuevos. Revista Española de Micropaleontología 28: 77-92.

Barreda, V.D. 1992. Muricingulisporis chenquensis, una nueva especie de espora de Pteridophyta del Terciario de Patagonia, Argentina. Ameghiniana 29: 347-351.

Barreda, V. 1997a. Palynomorph assemblage of the Chenque Formation, late Oligocene?-Miocene from Golfo San Jorge Basin, Patagonia, Argentina. Part 1: Gymnosperm and colpate pollen. Ameghiniana 34: 81-92.

Barreda, V. 1997b. Palynomorph assemblage of the Chenque Formation, late Oligocene?-Miocene from Golfo San Jorge Basin, Patagonia, Argentina. Part 3. Polycolpate and tricolporate pollen. Ameghiniana 34: 131-144.

Barreda, V. 1997c. Palynomorph assemblage of the Chenque Formation, late Oligocene?-Miocene from Golfo San Jorge Basin, Patagonia, Argentina. Part 4. Polycolporate and porate pollen. Ameghiniana 34: 145-154.

Barreda, V., Anzótegui, L.M., Prieto, A.R., Aceñolaza, P., Bianchi, M.M., Borromei, A.M., Brea, M., Caccavari, M., Cuadrado, G.A., Garralla, S., Grill, S., Guerstein, G.R., Lutz, A.I., Mancini, M.V., Mautino, L.R., Ottone, E.G., Quattrocchio, M., Romero, E.J., Zamaloa, M.C. y Zucol, A. 2007. Diversificación y cambios de las angiospermas durante el Neógeno en Argentina. En: S. Archangelsky, T. Sánchez y E.P. Tonni (Eds.), Ameghiniana 50 Aniversario. Asociación Paleontológica Argentina, Publicación Especial 11: 173-191.

Barreda, V.D. y Caccavari, M. 1992. Mimosoideae (Leguminosae) occurrences in the Early Miocene of Patagonia (Argentina). Palaeogeography, Palaeo- 
climatology, Palaeoecology 94: 243-252.

Barreda, V., Encinas, A. y Hinojosa, L.F. 2011. Polen y esporas de la Formación Navidad, Neógeno de Chile. Revista Chilena de Historia Natural 84: 341-355.

Barreda, V.D., Gutierrez, P.R. y Limarino, C.O. 1998. Edad y paleoambiente de la "Serie del Yeso", valle del Cura, provincia de San Juan: evidencias palinológicas. Ameghiniana 35: 321-335.

Barreda, V., Limarino, C., Fauqué, L., Tripaldi, A. y Net, L. 2003. Primer registro palinológico del miembro inferior de la Formación Cerro Morado (Mioceno), Precordillera de La Rioja. Ameghiniana 40: 81-87.

Barreda, V.D., Ottone, E.G., Dávila, F.M. y Astini, R.A. 2006. Edad y paleoambiente de la Formación del Buey (Mioceno), sierra de Famatina, La Rioja, Argentina: evidencias sedimentológicas y palinológicas. Ameghiniana 43: 215-226.

Bracco, A., León, L., Prámparo, M., Cerdeño, E. y Contreras, V. 2011. Stratigraphy and sedimentology of the Neogene deposits of Puchuzum, San Juan, Argentina. En: J.A. Salfity y R.A. Marquillas (Eds.), Cenozoic geology of the Central Andes of Argentina. SCS Publisher, Salta, p. 65-74.

Bremer, B., Bremer, K., Chase, M.W., Fay, M.F., Reveal, J.L., Soltis, D. E., Soltis, P.S., Stevens, P.F., Anderberg, A.A., Moore, M.J., Olmstead, R.G., Rudall, P.J., Sytsma, K.J., Tank, D.C., Wurdack, K., Xiang, J.Q.Y. y Zmarzty, S. 2009. An update of the Angiosperm Phylogeny Group classification for the orders and families of flowering plants: the APG III. Botanical Journal of the Linnean Society 161: 105-121.

Boltovskoy, E. 1979. Paleooceanografía del Atlántico sudoccidental desde el Mioceno, según estudios foraminiferológicos. Ameghiniana 16: 357-389.

Buchheim, M., Buchheim, J., Carlson, T., Braband, A., Hepperle, D., Krienitz, L., Wolf, M. y Egewald, E. 2005. Phylogeny of the Hydrodictyaceae (Chlorophyceae): inferences from rDNA data. Journal of Phycology 41: 1039-1054

Christenhusz, M.J.M., Reveal, J.L., Farjon, A., Gardner, M.F., Mill, R.R. y Chase, M.W. 2011. A new classification and linear sequence of extant gymnosperms. Phytotaxa 19: 55-70.

Cione, A.L. 1986. Los peces continentales del Cenozoico de Argentina. Su significación paleoambiental y paleogeográfica. $4^{\circ}$ Congreso Argentino de Paleontología y Bioestratigrafía (Mendoza), Actas 2: 101-106.

Cione, A.L., Vergani, G., Starck, D. y Herbst, R. 1995. Los peces del Mioceno de la Quebrada de la Yesera, provincia de Salta, Argentina. Su valor como indicadores ambientales y su antigüedad. Ameghiniana 32: 129-140.

Collinson, M.E. 2002. The ecology of Cainozoic ferns. Review of Palaeobotany and Palynology 119: 51-68.

Cookson, I.C. 1947. Plant microfossils from the lignites of Kerguelen Archipielago. British, Australian and New Zealand Antarctic Research Expedition 1929-31 Reports, Series A 2: 127-142.

Corda, A.J.C. 1839. Observations sur les Euastrées et les Cosmariées. Almanach de Carlsbad 9: 213-244.

Couper, R.A. 1953. Upper Mesozoic and Cainozoic spores and pollen grains from New Zealand. New Zealand Geological Survey Paleontological Bulletin 22, Wellington, 77 p.

Crandall-Stotler, B. y Stotler, R.E. 2000. Morphology and classification of the Marchantiophyta. En: A. J. Shaw y B. Goffinet (Eds.), Bryophyte biology. Cambridge University Press, Cambridge, p. 21-70.

Cuadrado, G.A. y Anzótegui, L.M. 1992. Resultados preliminares del análisis palinológico de la Formación Palo Pintado, Mioceno de Salta, Argentina. Asociación Paleontológica Argentina, Publicación Especial 2: 51-53.

Frederiksen, N.O. 1985. Review of Early Tertiary sporomorph paleoecology. American Association of Stratigraphic Palynologists Foundation, Contributions Series 15, Dallas, $92 \mathrm{p}$.

Galli, C.I., Anzótegui, L.M., Horn, M.Y. y Morton, L.S. 2011. Paleoambiente y paleocomunidades de la Formación Palo Pintado (Mioceno-Plioceno), provincia de Salta, Argentina. Revista Mexicana de Ciencias Geológicas 28: 161-174.

Garcia, M.J., Bistrichi, C.A., Saad, A.R., Alves Campanha, V. y De Oliveira, P.E. 2008. Stratigraphy and palaeoenvironments of the Tanque Basin, southeastern Brazil. Revista Brasileira de Paleontologia 11: 147-168.

Gassmann, M.I. y Pérez, C.F. 2006a. El modelado del transporte polínico de larga distancia en el sudeste de la provincia de Buenos Aires, Mar del Plata (Argentina): Celtis y Nothofagus, dos casos de estudio. $13^{\circ}$ Simposio Ar- gentino de Paleobotánica y Palinología (Bahía Blanca), Resúmenes: 106.

Gassmann, M.I. y Pérez, C.F. 2006b. Trajectories associated to regional and extra-regional pollen transport in the southeast of Buenos Aires province, Mar del Plata (Argentina). International Journal of Biometeorology 50: 280-291.

Gavriloff, I.J.C. y Arce, M.N. 2010. The presence of foraminifera in lacustrine facies of the Santa María Group (San José and Chiquimil Formations), middle/ upper Miocene, Tucumán and Catamarca, Argentina. $10^{\circ}$ Congreso Argentino de Paleontología y Bioestratigrafía y $7^{\circ}$ Congreso Latinoamericano de Paleontología (La Plata), Resúmenes: 91-92.

Gemeraad, J.J., Hopping, G.A. y Müller, J. 1968. Palynology of Tertiary sediments from tropical areas. Review of Palaeobotany and Palynology 6: 189-348.

González, M.A., Musacchio, E.A., García, A., Pascual, R. y Corte, A.H. 1980. Sobre la presencia de foraminíferos en sedimentos holocenos de la Salina del Bebedero (San Luis, Argentina). Simposio sobre problemas geológicos del litoral atlántico bonaerense (Mar del Plata), Resúmenes: 253-269.

Hernández, R.M., Jordan, T.E., Dalenz Farjat, A., Echavarría, L., Idleman, B.D. y Reynolds, J.H. 2005. Age, distribution, tectonics, and eustatic controls of the Paranense and Caribbean marine transgressions in southern Bolivia and Argentina. Journal of South American Earth Sciences 19: 495-512.

Heywood, V.H. 1993. Flowering plants of the world. Oxford University Press, New York, 335 p.

Knapp, M., Stöckler, K., Havell, D., Delsuc, F., Sebastianini, F. y Lockhart, P.J. 2005. Relaxed molecular clock provides evidence for long-distance dispersal of Nothofagus (southern beech). Plos Biology 3: 38-43.

Kofoid, C.A. 1911. Dinoflagellata of the San Diego region, IV. The genus Gonyaulax, with notes on its skeletal morphology and a discussion on its generic and specific characters. University of California Publications on Zoology 8: 187-286.

Komárek, J. y Janovská, V. 2001. Review of the green algal genus Pediastrum; implications for pollen-analytical research. Bibliotheca Phycologica, Band 108, Stuttgart, $127 \mathrm{p}$.

Komárek, J. y Marvan, P. 1992. Morpholopgical differences in natural populations of the genus Botryococcus (Chlorophyceae). Archive für Protistenkunde 141: 65-100.

Kouli, K., Birnkhuis, H. y Dale B. 2001. Spiniferites cruciformis: a fresh water dinoflagellate cyst? Review of Palaeobotany and Palynology 113: 273-286.

Laprida, C., Orgeira, M.J. y García Chapori, N. 2009. El registro de la Pequeña Edad de Hielo en lagunas pampeanas. Revista de la Asociación Geológica Argentina 65: 603-611.

Latorre, F., Pérez, C.F., Stutz, S. y Pastorino, S. 2010. Pollen deposition in Tauber traps and surface soil samples in the Mar Chiquita coastal lagoon area, pampa grasslands (Argentina). Boletín de la Sociedad Argentina de Botánica 45: 321-332.

Malumián, N. 1970. Estratigrafía del Terciario marino del subsuelo de la provincia de Buenos Aires (Argentina). Ameghiniana 7: 173-204.

Majas, F.D. y Romero, E.J. 1992. Aeropalynological research in the Northeast of Buenos Aires Province, Argentina. Grana 31: 143-156.

Marengo, H.G. 2006. [Micropaleontología y estratigrafia del Mioceno marino de la Argentina: las transgresiones de Laguna Paiva y del " Entrerriense-Paranense". Universidad de Buenos Aires, Tesis Doctoral, 122 p. Inédito].

Markgraf, V. 1983. Late and postglacial vegetational and paleoclimatic changes in subantarctic, temperate, and arid environments in Argentina. Palynology 7: 43-70.

Martin, H.A. 1989. Vegetation and climate of the late Cainozoic in the Murray Basin and their bearing on the salinity problem. Journal of Australian Geology and Geophysics 11: 291-299.

Martin, H.A. 2000. Re-assignment of the affinities of the fossil pollen type Tricolpites trioblatus Mildenhall and Pocknall to Wilsonia (Convolvulaceae) and a reassessment of the ecological interpretations. Review of Palaeobotany and Palynology 111: 237-251.

Mautino, L.R. 2007. Chlorophyta de los Valles Calchaquíes (Mioceno Medio y Superior), Argentina. Revista Española de Micropaleontología 39: 81102.

Mautino, L.R. 2011. Nuevas especies de palinomorfos de las formaciones San José y Chiquimil (Mioceno Medio y Superior), noroeste de Argentina. Re- 
vista Brasileira de Paleontologia 14: 279-290.

Mautino, L.R. y Anzótegui, L.M. 1998. Palinología de la Formación Chiquimil (Mioceno Superior) en Río Vallecito, provincia de Catamarca, Argentina. Parte I. Esporas: especies nuevas. Ameghiniana 35: 227-233.

Mautino, L.R. y Anzótegui, L.M. 2000. Esporas del Mioceno y retrabajadas del Mesozoico en la Formación Chiquimil, Vallecito, provincia de Catamarca. Ameghiniana 37: 13-22.

Mautino, L.R. y Anzótegui, L.M. 2002a. Palinología de la Formación Chiquimil (Mioceno Superior), en Río Vallecito, provincia de Catamarca, Argentina. Parte 2. Polen. Ameghiniana 39: 257-270.

Mautino, L.R. y Anzótegui, L.M. 2002b. Palinología de la Formación Chiquimil (Mioceno Superior), en Río Vallecito, provincia de Catamarca, Argentina. Parte 3. Polen. Ameghiniana 39: 271-284.

Mautino, L.R., Anzótegui, L., Garralla, S. y Herbst, R. 2012. Paleoflora de la Formación San José (Mioceno Medio) en la localidad Tolombón (Valle de Santa María), provincia de Salta. $15^{\circ}$ Simposio Argentino de Paleobotánica y Palinología (Corrientes), Resúmenes: 81.

Mautino, L.R., Cuadrado, G.A. y Anzótegui, L.M. 2004. Novedades taxonómicas, diversidad y significado evolutivo del polen de Malvaceae en el Terciario de Argentina. Revista Española de Micropaleontología 36: 467483

Menéndez, C.A. y Caccavari de Fílice, M.A. 1975. Las especies de Nothofagidites (polen fósil de Nothofagus) de sedimentos terciarios y cretácicos de Estancia La Sara, norte de Tierra del Fuego. Ameghiniana 12: 165-183.

Mildenhall, D.C. 1972. Fossil pollen of Acacia type from New Zealand. New Zealand Journal of Botany 10: 485-494.

Mildenhall, D.C. y Pocknall, D.T. 1989. Miocene-Pleistocene spores and pollen from Central Otago, South Island, New Zealand. New Zealand Geological Survey Paleontological Bulletin 89: 1-128.

Morton, L.S. 1984. Corbiculidae (Pelecípoda) de la Formación San José (Mioceno Superior) del Valle de Santa María, provincias de Catamarca y Tucumán, Argentina. Facena 5: 41-55.

Morton, L.S. 1986. Gastrópodos de las formaciones San José y Chiquimil (Mioceno Tardío) Catamarca y Tucumán, Argentina. Ameghiniana 23: 203-211.

Nitiu, D.S. 2009. Estudio del polen atmosférico y su relación con la vegetación local. La Plata, Argentina. Acta Botánica Malacitana 34: 189-199.

Ottone, E.G., Barreda, V.D. y Pérez, D.J. 1998. Basin evolution as reflected by Miocene palynomorphs from the Chinches Formation, Frontal Cordillera $\left(32^{\circ} \mathrm{S}\right)$, San Juan Province, Argentina. Revista Española de Micropaleontología 30: 35-47.

Pérez, D.J., Ottone, E.G. y Ramos, V.A. 1996. La ingresión marina miocena en la provincia de San Juan: sus implicancias paleogeográficas. $13^{\circ}$ Congreso Geológico Argentino y $3^{\circ}$ Congreso de Exploración de Hidrocarburos (Buenos Aires), Actas 1: 385-398.

Prámparo, M.B., Papú, O.H. y Contreras, V. 1998. Primer registro polínico en el Neógeno de la localidad de Puchuzum, provincia de San Juan, Argentina. $7^{\circ}$ Congreso Argentino de Paleontología y Bioestratigrafía (Bahía Blanca), Resúmenes: 27.

Prámparo, M.B., Papú, O.H. y Milana, J.P. 1996. Estudio palinológico del Miembro Inferior de la Formación Pachaco, Terciario de la provincia de San Juan. Descripciones sistemáticas. Ameghiniana 33: 397-407.

Quattrocchio, M., Durango de Cabrera, J. y Galli, C. 2003. Formación Anta (Mioceno Temprano/ Medio), Subgrupo Metán (Grupo Orán), en el río Piedras, pcia. de Salta. Datos palinológicos. Revista de la Asociación Geológica Argentina 58: 117-127.

Ramos, V.A. y Alonso, R.N. 1995. El mar Paranense en la provincia de Jujuy. Revista del Instituto de Geología y Minería 10: 73-80.

Reveal, J.L. 2012. An outline of a classification scheme for extant flowering plants. Phytoneuron 37: 1-221.

Richards, P.W. 1998. The tropical rain forest. Second Edition. Cambridge University Press, Cambridge, $575 \mathrm{p}$

Rochon, A., Lewis, J., Ellegaard, M. y Harding, I.C. 2009. The Gonyaulax spinifera (Dinophyceae) "complex"': perpetuating the paradox? Review of Palaeobotany and Palynology 155: 52-60.
Romero, E.J. 1973. Polen fósil de Nothofagus (Nothofagidites) del Cretácico y Paleoceno de Patagonia. Revista del Museo de La Plata, nueva serie, 7, Paleontología 47: 291-303.

Romero, E.J. 1977. Polen de gimnospermas y fagáceas de la Formación Río Turbio (Eoceno), Santa Cruz, Argentina. Fundación para la Educación, la Ciencia y la Cultura, Buenos Aires, 219 p.

Ruskin, B.G., Dávila, F.M., Hoke, G.D., Jordan, T.E., Astini, R.A. y Alonso, R. 2011. Stable isotope composition of middle Miocene carbonates of the Frontal Cordillera and Sierras Pampeanas: Did the Paranaense seaway flood western and central Argentina? Palaeogeography, Palaeoclimatology, Palaeoecology 308: 293-303.

Russo, A. y Serraiotto, A. 1978. Contribución al conocimiento de la estratigrafía terciaria en el noroeste argentino. $7^{\circ}$ Congreso Geológico Argentino (Neuquén), Actas 1: 715-730.

Scasso, R.A., McArthur, J.M., del Río, C.J., Martínez, S. y Thirlwall, M.F. 2001. ${ }^{87} \mathrm{Sr} /{ }^{86} \mathrm{Sr}$ Late Miocene age of fossil molluscs in the 'Entrerriense' of the Valdés Peninsula (Chubut, Argentina). Journal of South Amnerican Earth Sciences 14: 319-329.

Smith, A.R., Pryer, K.M., Schuettpelz, E., Korall, P., Schneider, H. y Wolf, P.G. 2006. A classification for extant ferns. Taxon 55: 705-731.

Soltis, D.E., Soltis, P.S., Endress, P.K. y Chase, M.W. 2005. Phylogeny and evolution of angiosperms. Sinauer Associates, Sunderland, 370 p.

Stancliffe, R.P.W. 1996. Microforaminiferal linings. En: J. Jansonius y D.C. McGregor (Eds.), Palynology: principles and applications. American Association of Stratigraphic Palynologists Foundation 2, College Station, p. 767-778.

Starck, D. y Anzótegui, L.M. 2001. The late miocene climatic change-persistence of a climatic signal through the orogenic stratigraphic record in northwestern Argentina. Journal of South American Earth Sciences 14: 763-774.

Stover, L.E. y Evans, P.R. 1973. Upper Cretaceous spore-pollen zonation, offshore Gippsland Basin, Australia. Geological Society of Australia, Special Publication 4: 55-72.

Torra, R. 2005. The Chaco Paraná Basin rift basin system. An approach to the tectonic-stratigraphical evolution from the Late Cretaceous to Quaternary. South America. Ciência e Natura, UFSM 27: 25-64.

Truswell, E.M., Sluiter, I.R. y Harris, W.K. 1985. Palynology of the Oligocene-Miocene sequences in the Oakvale 1 corehole, western Murray Basin, South Australia. Journal of Australian Geology and Geophisics 9: 267-295.

Wall, D., Dale, B. y Harada, K. 1973. Descriptions of new fossil dinoflagellates from the Late Quaternary of the Black Sea. Micropaleontology 19: $18-31$.

Zabert, L.L. 1984. Nonion demens (Bik), foraminífero bentónico en el Mioceno del Valle de Santa María (Tucumán) y Selva (Santiago del Estero), Argentina. $3^{\circ}$ Congreso Argentino de Paleontología y Bioestratigrafía (Corrientes), Actas: 183-196.

Zamaloa, M.C. y Romero, E.J. 1990. Some spores and pollen from the Cullen Formation (upper Eocene to middle Oligocene), Tierra del Fuego, Argentina. Palynology 14: 123-133.

Zamora, N., Medeanic, S. y Corrêa, I.C.S. 2007. Microforaminíferos como indicadores paleoambientais: estudos palinológicos da região sul da Costa Rica, América Central. Gravel 5: 75-87.

doi: 10.5710/AMEGH.24.07.2013.603

Recibido: 09 de agosto de 2012

Aceptado: 24 de julio de 2013 